Tropical Journal of Pharmaceutical Research May 2019; 18 (5): 1123-1132

ISSN: $1596-5996$ (print); 1596-9827 (electronic)

(C) Pharmacotherapy Group, Faculty of Pharmacy, University of Benin, Benin City, 300001 Nigeria.

\title{
Hypertension-related knowledge, medication adherence and health-related quality of life (HRQoL) among hypertensive patients in Islamabad, Pakistan
}

\author{
Muhammad Amer ${ }^{1}$, Nisar-Ur-Rahman ${ }^{1}$, Saeed-Ur-Rashid Nazir ${ }^{2 \star}$, Atif Raza ${ }^{3}$, \\ Humayun Riaz ${ }^{4}$, Saleha Sadeeqa ${ }^{5}$, Misbah Sultana ${ }^{3}$ \\ ${ }^{1}$ Department of Pharmacy, COMSATS University Islamabad, Abbottabad Campus, ${ }^{2}$ Faculty of Pharmacy, University of \\ Sargodha, Sargodha, ${ }^{3}$ University College of Pharmacy, University of Punjab, ${ }^{4}$ Rashid Latif College of Pharmacy, ${ }^{5}$ Institute of \\ Pharmacy, Lahore College for Women University, Lahore, Pakistan
}

*For correspondence: Email: srnazir@yahoo.com; Tel: +92-301-462-9275

Sent for review: 10 October 2018

Revised accepted: 17 April 2019

\begin{abstract}
Purpose: To determine knowledge regarding hypertension, adherence to medication and HealthRelated Quality of Life (HRQoL), and their associations in hypertensive patients in Pakistan.

Methods: A cross-sectional study was conducted among 384 hypertensive patients attending a tertiary health care public sector hospital in Islamabad, Pakistan. Data were collected using knowledge questionnaire regarding hypertension, Morisky Medication Adherence Scale, and EuroQol (EQ-5D) scale.

Results: The mean systolic and diastolic blood pressures of the 384 patients were $140.39 \pm 15.485$ and $88.74 \pm 10.683 \mathrm{mmHg}$, respectively. The coefficient of correlation between knowledge regarding hypertension and adherence was $0.638(p<0.001)$, showing a positive association. The correlation coefficient between knowledge and HRQoL was 0.709 ( $p<0.001)$, suggesting a good association. The correlation coefficient between adherence to medication and HRQoL was 0.545 ( $p<0.001)$, which indicated a positive correlation.

Conclusion: These results indicate that there are statistically significant associations between hypertension knowledge and HRQoL, hypertension knowledge and medication adherence, and between adherence and HRQoL in the hypertensive patients studied.
\end{abstract}

Keywords: Hypertension, Knowledge, Medication adherence, Blood pressure

\begin{abstract}
This is an Open Access article that uses a fund-ing model which does not charge readers or their institutions for access and distributed under the terms of the Creative Commons Attribution License (http://creativecommons.org/licenses/by/4.0) and the Budapest Open Access Initiative (http://www.budapestopenaccessinitiative.org/read), which permit unrestricted use, distribution, and reproduction in any medium, provided the original work is properly credited.

Tropical Journal of Pharmaceutical Research is indexed by Science Citation Index (SciSearch), Scopus, International Pharmaceutical Abstract, Chemical Abstracts, Embase, Index Copernicus, EBSCO, African Index Medicus, JournalSeek, Journal Citation Reports/Science Edition, Directory of Open Access Journals (DOAJ), African Journal Online, Bioline International, Open-J-Gate and Pharmacy Abstracts
\end{abstract}

\section{INTRODUCTION}

Hypertension (HTN) is a chronic disease of worldwide concern. It is an important and avoidable risk factor for cardiovascular diseases. Hypertension has substantial negative impacts on health, and results in needless morbidity and mortality. It is believed to be responsible for $>5.8$ $\%$ of deaths worldwide, and loss of about $11.9 \%$ of life expectancy. The success rate in control of hypertension is poor all over the world. Pakistan is faced with an epidemic of hypertension as well as cardiovascular (CV) diseases [1]. Life expectancy in Pakistan (62 years) is $17-20$ 
years lower than the range in developed countries [2]. Low knowledge regarding hypertension as well as other cardiovascular diseases has been reported on the continent.

Data relating to knowledge as well as risk factors related to hypertension are not available in Pakistan. This has become a major public health and clinical issue in Pakistan. It has been reported that the prevalence of hypertension in Pakistan is $33 \%$. Every third individual aged above forty years is prone to different diseases. It has also been reported that only $50 \%$ of hypertensive individuals were diagnosed, while only about half of the diagnosed patients received treatment. Half of those diagnosed patients treated for hypertension were prescribed with correct medication for effective control of hypertension. Thus, only $12.5 \%$ of hypertension cases were properly controlled [1].

The present study was aimed at assessing the level of hypertension-related knowledge, adherence to medication and HRQoL among hypertensive patients in Islamabad, Pakistan and to assess the association between hypertension related knowledge, medication adherence and HRQoL.

\section{METHODS}

\section{Study design and settings}

A questionnaire-based, cross sectional study was adopted to assess knowledge, HRQoL and blood pressure of hypertensive patients. Since the prevalence of hypertension is $33 \%$ in Pakistan, a sample of 384 patients, based on prevalence [1] was recruited from Federal Government Poly Clinic (Postgraduate Medical Institute). The study was conducted from August to November 2017.

\section{Inclusion criteria}

Patients aged thirty years and above, who were diagnosed with hypertension, and were receiving treatment for high blood pressure in the previous 6-months, and patients with the ability to write or speak Urdu (Pakistan's official language) were recruited for this study.

\section{Exclusion criteria}

Patients aged less than thirty years, and those aged over seventy years; pregnant women, patients with co-morbidities, patients having dementia, and immigrants were excluded from this study.

\section{Ethical approval}

Approval for this study was obtained from the Ethical Committee of Poly Clinic Hospital (affiliated to Shaheed Zulfiqar Ali Bhutto Medical University, Islamabad) (approval no. FGPC.1/12/2016/Ethical Committee). The study was conducted according to ethical principles as described in Helsinki Declaration of 1964, revised in 2013 [3]. Prior to collection of data, written consent was obtained from the hypertensive patients.

\section{Data collection}

Knowledge questionnaire about hypertension, Morisky medication adherence scale (MMAS-U) and EuroQol (EQ-5D) scale were utilized for data collection.

\section{Assessment of knowledge of hypertension}

Hypertension knowledge questionnaire in Urdu was developed after extensive survey of literature [4]. It consisted of 22 questions. Each question required a 'Yes', 'No' or 'Do Not Know' response. Knowledge was evaluated by scoring 1 for a correct response, and 0 for a wrong response. The response of 'Do Not Know' was also scored zero. Knowledge was measured on a scale from a minimum of 0 to maximum of 22 . Scores < 10 were categorized as poor, while scores in the ranges of $10-18$ and $19-22$ were categorized as moderate and adequate knowledge, respectively, about hypertension.

\section{Assessment of medication adherence}

The Urdu version of Medication Adherence questionnaire by Morisky was used to evaluate adherence to treatment. It included seven questions having responses of Yes or No, and 1 question as a 5-point Likert type scale. Each "No" response was scored 1, and each "Yes" response scored 0 except for question 5, in which each "Yes" response was scored 1. Adherence scores were grouped viz: 8 for high adherence, 6 - 7 for medium adherence, and $<6$ for low adherence [5].

\section{Assessment of HRQoL}

The HRQoL was measured in hypertensive patients using EuroQol EQ-5D scale which included 2 parts [6]. The first part comprised 5 dimensions of health: mobility, self-care, usual activities, pain/discomfort, and anxiety/depression. Each dimension had three levels viz: no problem, some or moderate 
problems and extreme problem corresponding to levels 1, 2, and 3, respectively [7].

For Pakistani population, EQ-5D preference weight was not available for each state of health. Therefore, as EQ-5D Index score, these preference weights were derived from Time Trade-Off (TTO) tariff of preference weights of UK general population [8]. The second part consisted of visual analogue scale (VAS). It determined the respondent's self-rated status of health on a graduated $(0-100)$ scale, with a score of 100 as the best imaginable state of health, and a score of 0 as the worst imaginable state of health, and higher scores for higher HRQoL. The Urdu version of EQ-5D was provided on request, by EuroQol. This study was also registered with EuroQol.

\section{Blood pressure measurement}

The blood pressure of each patient was measured using a calibrated mercury sphygmomanometer.

\section{Data analysis}

Data were analyzed statistically using SPSS software version 21.0. Spearman's test was performed to assess any association between knowledge, medication adherence and HRQoL. Measurement data are expressed as mean \pm standard deviation (SD).

\section{RESULTS}

\section{Patients' demographics}

Cronbach's alpha value was 0.755 for hypertension knowledge, 0.747 for adherence, and 0.712 for EQ-5D questionnaires. Three hundred and eighty-four (384) HTN patients were enrolled in this study. Table 1 shows the sociodemographic variables as well as frequency distribution of the hypertensive patients.

Table shows that 138 out of the 384 patients were in the age range of 41 - 50 years, with $56 \%$ as males. Three hundred and eighty-three of the patients $(99.7 \%)$ were married. Mean duration of disease was $3.31 \pm 2.13$ years. Majority of the patients $(101,26.3 \%)$ had matriculation level of education, while 156 patients $(40.6 \%)$ were either housewives or house makers. Two hundred and twenty-one patients $(57.6 \%)$ had a monthly income of more than 15,000 Pakistan Rupees, and 220 patients $(57.3 \%)$ were urban dwellers.
Table 1: Demographic characteristics of survey respondents $(n=384)$

\begin{tabular}{|c|c|c|}
\hline Characteristic & Frequency & (\%) \\
\hline \multicolumn{3}{|l|}{ Age (years) } \\
\hline $30-40$ & 61 & 15.9 \\
\hline $41-50$ & 138 & 35.9 \\
\hline $51-60$ & 125 & 32.6 \\
\hline $61-70$ & 60 & 15.6 \\
\hline \multicolumn{3}{|l|}{ Gender } \\
\hline Male & 215 & 56 \\
\hline Female & 169 & 44 \\
\hline \multicolumn{3}{|l|}{ Marital status } \\
\hline Married & 383 & 99.7 \\
\hline Unmarried & 0 & 0.00 \\
\hline Widow & 1 & 0.30 \\
\hline \multicolumn{3}{|l|}{ Education } \\
\hline Illiterate & 87 & 22.7 \\
\hline Primary & 37 & 9.6 \\
\hline Middle & 58 & 15.1 \\
\hline Matriculation & 101 & 26.3 \\
\hline Intermediate & 29 & 7.6 \\
\hline Graduate & 47 & 12.2 \\
\hline Postgraduate & 25 & 6.5 \\
\hline \multicolumn{3}{|l|}{ Occupation } \\
\hline Private sector job & 47 & 12.2 \\
\hline Government job & 121 & 31.5 \\
\hline Businessman & 0 & 0.00 \\
\hline Housewife/Housemaker & 156 & 40.6 \\
\hline Retired & 60 & 15.6 \\
\hline Jobless & 0 & 0.00 \\
\hline \multicolumn{3}{|c|}{ Monthly income ${ }^{*}$ (Pakistan rupees) } \\
\hline Nil & 157 & 40.9 \\
\hline $5000-10000$ & 2 & 0.50 \\
\hline $10001-15001$ & 4 & 1.00 \\
\hline$>15001$ & 221 & 57.6 \\
\hline \multicolumn{3}{|l|}{ Location } \\
\hline Urban & 220 & 57.3 \\
\hline Rural & 164 & 42.7 \\
\hline \multicolumn{3}{|c|}{$\begin{array}{l}\text { Duration of disease (years, mean } \pm S D \text { ) }=3.31 \pm \\
2.13\end{array}$} \\
\hline$<1$ & 80 & 20.8 \\
\hline $1-3$ & 164 & 42.7 \\
\hline $3-5$ & 26 & 6.8 \\
\hline$>5$ & 114 & 29.7 \\
\hline
\end{tabular}

${ }^{*} \mathrm{US} \$ 1=111.70 \mathrm{Pk}$ Rs

\section{Blood pressure}

Mean systolic and diastolic blood pressures of hypertensive patients $(n=384)$ were $140.39 \pm$ 15.485 and $88.74 \pm 10.683$ in $\mathrm{mmHg}$, respectively.

\section{Patients' knowledge about hypertension}

Table 2 shows responses of patients to hypertension knowledge questions. Poor knowledge was reported in responses to questions 4 (39.6 $\pm 20.8,60.4 \%), 7$ (55.2 \pm 1.0 , $56.2 \%), 8(78.9 \%), 10(88.3 \pm 2.3,90.6 \%), 13$ $(55.7 \pm 4.7,60.4 \%), \quad 15 \quad(66.4 \pm 1.0=67.4 \%), 16$ $(47.9 \pm 11.7,59.6 \%)$ and $22(39.6 \pm 16.4,56 \%)$. 
Correct answers to questions 6,13 and 15 were $58.1,39.6$ and 32.6 percent, respectively.

Table 2: Responses to hypertension knowledge questions

\begin{tabular}{|c|c|c|c|}
\hline Knowledge questionnaire & $\begin{array}{l}\text { Yes } \\
(\%)\end{array}$ & $\begin{array}{l}\text { No } \\
(\%)\end{array}$ & $\begin{array}{c}\text { Don't } \\
\text { know } \\
(\%)\end{array}$ \\
\hline $\begin{array}{l}\text { 1. Do you know the normal } \\
\text { values of blood pressure }\end{array}$ & 72.7 & 26.0 & 1.3 \\
\hline $\begin{array}{l}\text { 2. Elevated blood pressure is } \\
\text { called hypertension }\end{array}$ & 76.8 & 14.1 & 9.1 \\
\hline $\begin{array}{l}\text { 3. Do you know the signs and } \\
\text { symptoms of hypertension? }\end{array}$ & 79.2 & 20.8 & 0.0 \\
\hline 4. Hypertension is hereditary & 39.6 & 39.6 & 20.8 \\
\hline $\begin{array}{l}\text { 5. The older a person is, the } \\
\text { greater their risk of having } \\
\text { hypertension }\end{array}$ & 65.1 & 23.2 & 11.7 \\
\hline $\begin{array}{l}\text { 6. Both men and women have } \\
\text { equal chance of developing } \\
\text { hypertension }\end{array}$ & 30.2 & 58.1 & 11.7 \\
\hline $\begin{array}{l}\text { 7. Do you know the names of } \\
\text { your prescribed drugs? }\end{array}$ & 43.8 & 55.2 & 1.0 \\
\hline $\begin{array}{l}\text { 8. Do you know the dose of } \\
\text { your medication? }\end{array}$ & 21.1 & 78.9 & 0.0 \\
\hline $\begin{array}{l}\text { 9. Do you know the frequency } \\
\text { of your medication? }\end{array}$ & 96.9 & 1.8 & 1.3 \\
\hline $\begin{array}{l}\text { 10. Do you know the possible } \\
\text { side effects of your } \\
\text { medicines? }\end{array}$ & 9.4 & 88.3 & 2.3 \\
\hline $\begin{array}{l}\text { 11. Smoking is a risk factor for } \\
\text { hypertension }\end{array}$ & 49.5 & 3.9 & 46.6 \\
\hline $\begin{array}{l}\text { 12. Being overweight increases } \\
\text { risk for hypertension }\end{array}$ & 81.2 & 18.2 & 0.6 \\
\hline $\begin{array}{l}\text { 13. White meat is as good as } \\
\text { red meat in hypertension }\end{array}$ & 55.7 & 39.6 & 4.7 \\
\hline $\begin{array}{l}\text { 14. Hypertension can lead to } \\
\text { other life-threatening } \\
\text { diseases. }\end{array}$ & 51.0 & 30.5 & 18.5 \\
\hline $\begin{array}{l}\text { 15. Medication alone can } \\
\text { control hypertension }\end{array}$ & 66.4 & 32.6 & 1.0 \\
\hline $\begin{array}{l}\text { 16. Dietary approaches can } \\
\text { help to reduce hypertension }\end{array}$ & 40.4 & 47.9 & 11.7 \\
\hline $\begin{array}{l}\text { 17. Salt reduction can control } \\
\text { hypertension }\end{array}$ & 81.5 & 17.4 & 1.0 \\
\hline $\begin{array}{l}\text { 18. Eating fatty food affects } \\
\text { blood cholesterol level } \\
\text { which is a risk factor for } \\
\text { developing hypertension }\end{array}$ & 95.3 & 4.2 & 0.5 \\
\hline $\begin{array}{l}\text { 19. Hypertension is a treatable } \\
\text { condition }\end{array}$ & 65.1 & 20.8 & 14.1 \\
\hline $\begin{array}{l}\text { 20. Stress can cause } \\
\text { hypertension }\end{array}$ & 99.0 & 0.5 & 0.5 \\
\hline $\begin{array}{l}\text { 21. Regular physical activity will } \\
\text { lower a person's chance of } \\
\text { getting hypertension }\end{array}$ & 85.9 & 9.4 & 4.7 \\
\hline $\begin{array}{l}\text { 22. Hypertension is a condition } \\
\text { which can progress with age }\end{array}$ & 44.0 & 39.6 & 16.4 \\
\hline
\end{tabular}

\section{Knowledge scores}

Table 3 shows knowledge scores of patients. The mean knowledge score was $13.26 \pm 5.16$ and median score was 13 . It also indicates the relationship between demographic characteristics and knowledge. Differences were statistically significant when age, gender, education, occupation and monthly income were analyzed $(p<0.001)$.

\section{Hypertension knowledge scale}

The result showed that 103 patients $(26.8 \%)$ with mean systolic BP of $145 \pm 15 \mathrm{mmHg}$, and mean diastolic BP of $91 \pm 13 \mathrm{mmHg}$ had poor knowledge score $(<10)$; 191 patients $(49.7 \%)$ with mean systolic BP of $141 \pm 16 \mathrm{mmHg}$ and mean diastolic BP of $90 \pm 9 \mathrm{mmHg}$, had moderate knowledge score (10 - 18), while 90 patients $(23.4 \%)$ with mean systolic BP (133 \pm $14 \mathrm{mmHg})$ and mean diastolic BP $(84 \pm 9 \mathrm{mmHg})$ had adequate knowledge score (19 - 22) about hypertension.

\section{Medication adherence}

Table 4 shows responses of patients to medication adherence. Poor adherence to medication was assessed for questions 1 - 4 . The correct answers with highest responses to questions 5, 6 and 8 were 85.2, 64.8 and $62.0 \%$, respectively.

\section{Adherence scores}

Table 5 shows adherence scores of patients. The mean adherence score was $4.66 \pm 2.743$ and median score was 4 .

\section{Medication adherence scale}

The results showed that 204 patients (53.1\%) with mean systolic BP of $148 \pm 14 \mathrm{mmHg}$ and mean diastolic BP of $92 \pm 11 \mathrm{mmHg}$, had low adherence score $(<6)$; 91 patients $(23.7 \%)$ with mean systolic BP $(135 \pm 12)$ and mean diastolic BP $(86 \pm 8)$ had medium adherence score $(6-7)$, while 89 patients $(23.2 \%)$ patients with mean systolic BP $(128 \pm 11 \mathrm{mmHg})$ and mean diastolic BP $(83 \pm 9 \mathrm{mmHg})$ had high adherence score (8).

\section{EQ-5D health status}

Table 6 shows the HRQoL scores of patients. The mean score of EQ-5D was $0.6456 \pm 0.2317$, and EQ-VAS score was $66.83 \pm 5.832$. The Table also shows the relationship between HRQoL and the demographic characteristics.

The 15 different health states of EQ-5D described are shown in Table 7. 
Table 3: Description of Mean Knowledge Score

\begin{tabular}{|c|c|c|c|c|}
\hline Description & $\mathbf{N}$ & Mean knowledge Score & SD. & P-value* \\
\hline \multicolumn{5}{|l|}{ Age (years) } \\
\hline $30-40$ & 61 & 14.90 & 4.78 & \multirow[t]{4}{*}{$<0.001$} \\
\hline $41-50$ & 138 & 14.14 & 4.62 & \\
\hline $51-60$ & 125 & 13.18 & 5.80 & \\
\hline $61-70$ & 60 & 9.72 & 3.47 & \\
\hline \multicolumn{5}{|l|}{ Gender } \\
\hline Male & 215 & 14.92 & 5.08 & \multirow[t]{2}{*}{$<0.001$} \\
\hline Female & 169 & 11.15 & 4.45 & \\
\hline \multicolumn{5}{|l|}{ Marital status } \\
\hline Married & 383 & 13.25 & 5.16 & \multirow[t]{3}{*}{0.603} \\
\hline Unmarried & 0 & 00.00 & 0.00 & \\
\hline Widow & 1 & 13.00 & 0.00 & \\
\hline \multicolumn{5}{|l|}{ Education } \\
\hline Illiterate & 87 & 10.69 & 4.66 & \multirow{7}{*}{$<0.001$} \\
\hline Primary & 37 & 9.83 & 3.98 & \\
\hline Middle & 58 & 12.01 & 4.65 & \\
\hline Matriculation & 101 & 14.66 & 4.86 & \\
\hline Intermediate & 29 & 13.86 & 4.63 & \\
\hline Graduate & 47 & 16.44 & 4.27 & \\
\hline Postgraduate & 25 & 17.72 & 4.16 & \\
\hline \multicolumn{5}{|l|}{ Occupation } \\
\hline Private Job & 47 & 14.93 & 4.78 & \multirow[t]{6}{*}{$<0.001$} \\
\hline Government Job & 121 & 17.15 & 4.13 & \\
\hline Businessman & 0 & 00.00 & 0.00 & \\
\hline Housewife/House Maker & 156 & 10.98 & 4.29 & \\
\hline Retired & 60 & 09.98 & 4.29 & \\
\hline Jobless & 0 & 00.00 & 0.00 & \\
\hline \multicolumn{5}{|c|}{ Monthly income (Pakistan rupees) } \\
\hline Nil & 157 & 10.88 & 4.27 & \multirow[t]{4}{*}{$<0.001$} \\
\hline $5000-10000$ & 2 & 7.50 & 4.94 & \\
\hline $10001-15001$ & 4 & 12.50 & 5.92 & \\
\hline$>15001$ & 221 & 15.01 & 5.04 & \\
\hline \multicolumn{5}{|l|}{ Location } \\
\hline Urban & 220 & 13.40 & 5.20 & \multirow[t]{2}{*}{0.820} \\
\hline Rural & 164 & 13.04 & 5.10 & \\
\hline \multicolumn{5}{|c|}{ Duration of disease (years, $3.31 \pm 2.13$ ) } \\
\hline$<1$ & 80 & 13.98 & 5.06 & \multirow[t]{4}{*}{0.640} \\
\hline $1-3$ & 164 & 12.89 & 5.02 & \\
\hline $3-5$ & 26 & 14.07 & 5.37 & \\
\hline$>5$ & 114 & 13.09 & 5.35 & \\
\hline
\end{tabular}

${ }^{*}$ Chi-square test, SD = standard deviation

Table 4: Self-reported adherence responses of patients

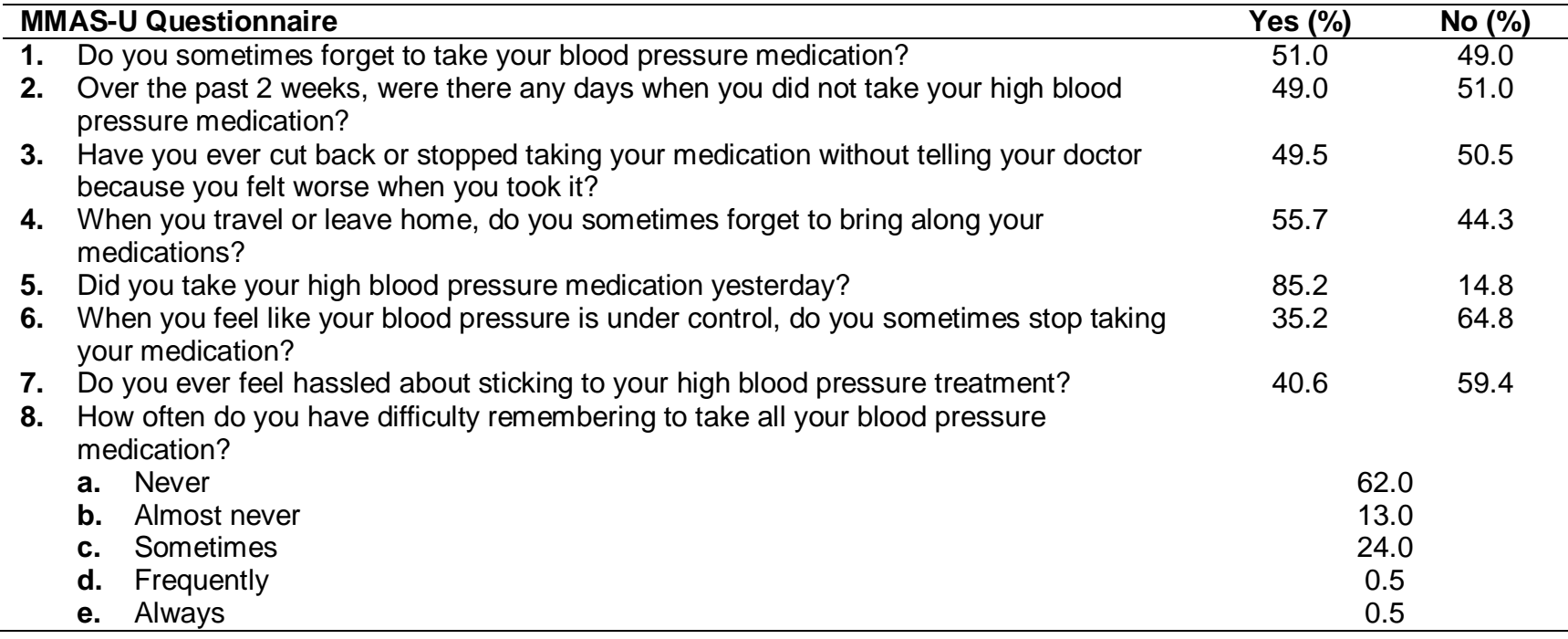


Table 5: Relationship between socio-demographic factors and mean adherence score

\begin{tabular}{|c|c|c|c|c|}
\hline Description & $\mathbf{N}$ & $\begin{array}{c}\text { Mean adherence } \\
\text { score }\end{array}$ & SD & $P$-value \\
\hline \multicolumn{5}{|l|}{ Age (year) $50.21 \pm 9.51$} \\
\hline $30-40$ & 61 & 4.93 & 2.74 & \multirow{4}{*}{0.028} \\
\hline $41-50$ & 138 & 5.02 & 2.65 & \\
\hline $51-60$ & 125 & 4.83 & 2.76 & \\
\hline $61-70$ & 60 & 3.17 & 2.49 & \\
\hline \multicolumn{5}{|l|}{ Gender } \\
\hline Male & 215 & 5.15 & 2.68 & \multirow{2}{*}{0.001} \\
\hline Female & 169 & 4.03 & 2.70 & \\
\hline \multicolumn{5}{|l|}{ Marital status } \\
\hline Married & 383 & 4.65 & 2.74 & \multirow{3}{*}{0.199} \\
\hline Unmarried & 0 & 00.00 & 00.00 & \\
\hline Widow & 1 & 7.00 & 00.00 & \\
\hline \multicolumn{5}{|l|}{ Education } \\
\hline Illiterate & 87 & 4.03 & 2.83 & \multirow{7}{*}{0.002} \\
\hline Primary & 37 & 3.97 & 2.62 & \\
\hline Middle & 58 & 4.38 & 2.80 & \\
\hline Matriculation & 101 & 5.20 & 2.66 & \\
\hline Intermediate & 29 & 4.28 & 2.66 & \\
\hline Graduate & 47 & 5.21 & 2.43 & \\
\hline Postgraduate & 25 & 5.68 & 2.85 & \\
\hline \multicolumn{5}{|l|}{ Occupation } \\
\hline Private job & 47 & 5.34 & 2.58 & \multirow{6}{*}{$<0.001$} \\
\hline Government job & 121 & 5.95 & 2.32 & \\
\hline Businessman & 0 & 00.00 & 00.00 & \\
\hline Housewife/Housemaker & 156 & 4.05 & 2.72 & \\
\hline Retired & 60 & 3.08 & 2.46 & \\
\hline Jobless & 0 & 00.00 & 00.00 & \\
\hline \multicolumn{5}{|c|}{ Monthly income (Pakistan rupees) } \\
\hline Nil & 157 & 3.99 & 2.72 & \multirow{4}{*}{0.032} \\
\hline $5000-10000$ & 2 & 5.50 & 0.71 & \\
\hline $10001-15001$ & 4 & 4.25 & 3.78 & \\
\hline$>15001$ & 221 & 5.13 & 2.67 & \\
\hline \multicolumn{5}{|l|}{ Location } \\
\hline Urban & 220 & 4.53 & 2.72 & \multirow{2}{*}{0.619} \\
\hline Rural & 164 & 4.82 & 2.77 & \\
\hline \multicolumn{5}{|c|}{ Duration of disease (year) $3.31 \pm 2.13$} \\
\hline$<1$ & 80 & 4.66 & 2.69 & \multirow{4}{*}{0.930} \\
\hline $1-3$ & 164 & 4.63 & 2.82 & \\
\hline $3-5$ & 26 & 5.00 & 2.88 & \\
\hline$>5$ & 114 & 4.61 & 2.68 & \\
\hline
\end{tabular}

\section{Association between knowledge and medication adherence}

The coefficient of correlation between knowledge related to hypertension and medication adherence was $0.638(p<0.001)$ which indicated positive correlation.

\section{Association between knowledge and HRQoL}

The coefficient of correlation between knowledge regarding hypertension and HRQoL was 0.709 ( $p$ $<0.001$ ) which indicated positive correlation. The coefficient of correlation between knowledge and VAS was $0.459(p<0.001)$ which also indicated positive correlation.

\section{Association between medication adherence and HRQoL}

The coefficient of correlation between adherence and HRQoL was $0.545(p<0.001)$ which indicated positive correlation. The coefficient of correlation between adherence and VAS was $0.328(p<0.001)$, which also indicated fair correlation. 
Table 6: Description of EQ-5D scores

\begin{tabular}{|c|c|c|c|c|c|c|}
\hline Description & $\mathbf{N}$ & $\begin{array}{l}\text { Mean EQ- } \\
\text { 5D Score }\end{array}$ & SD & EQ-VAS & SD & P-value \\
\hline \multicolumn{7}{|l|}{ Age (year) ${ }^{\star *}$} \\
\hline $30-40$ & 61 & 0.6754 & 0.2024 & 72.97 & 3.4540 & \multirow{4}{*}{$<0.001$} \\
\hline $41-50$ & 138 & 0.6773 & 0.2211 & 68.73 & 5.1920 & \\
\hline $51-60$ & 125 & 0.6337 & 0.2387 & 64.38 & 4.5630 & \\
\hline $61-70$ & 60 & 0.5669 & 0.2525 & 61.30 & 3.3260 & \\
\hline \multicolumn{7}{|l|}{ Gender* } \\
\hline Male & 215 & 0.6910 & 0.1725 & 66.68 & 5.1760 & \multirow{2}{*}{$<0.001$} \\
\hline Female & 169 & 0.5877 & 0.2801 & 67.01 & 6.5850 & \\
\hline \multicolumn{7}{|l|}{ Marital status ${ }^{* *}$} \\
\hline Married & 383 & 0.6453 & 0.2320 & 66.82 & 5.8380 & \multirow{3}{*}{0.765} \\
\hline Unmarried & 0 & 0.0000 & 0.0000 & 00.00 & 0.0000 & \\
\hline Widow & 1 & 0.7250 & 0.0000 & 70.00 & 0.0000 & \\
\hline \multicolumn{7}{|l|}{ Education** } \\
\hline Illiterate & 87 & 0.60818 & 0.2854 & 66.17 & 6.3600 & \multirow{7}{*}{0.036} \\
\hline Primary & 37 & 0.5962 & 0.2651 & 65.41 & 6.7020 & \\
\hline Middle & 58 & 0.5898 & 0.2748 & 65.36 & 5.9520 & \\
\hline Matriculation & 101 & 0.7005 & 0.1494 & 68.39 & 4.8850 & \\
\hline Intermediate & 29 & 0.6290 & 0.2455 & 68.28 & 6.0290 & \\
\hline Graduate & 47 & 0.6841 & 0.1664 & 66.68 & 5.3490 & \\
\hline Postgraduate & 25 & 0.7032 & 0.1676 & 66.88 & 5.1990 & \\
\hline \multicolumn{7}{|l|}{ Occupation** } \\
\hline Private job & 47 & 0.6706 & 0.2167 & 67.72 & 5.5510 & \multirow{6}{*}{$<0.001$} \\
\hline $\begin{array}{l}\text { Government } \\
\text { job }\end{array}$ & 121 & 0.7357 & 0.1159 & 68.66 & 4.6000 & \\
\hline Businessman & 0 & 0.0000 & 0.0000 & 00.00 & 0.0000 & \\
\hline $\begin{array}{l}\text { Housewife/ } \\
\text { Housemaker }\end{array}$ & 156 & 0.5872 & 0.2810 & 67.18 & 6.4160 & \\
\hline Retired & 60 & 0.5960 & 0.2202 & 61.50 & 2.9770 & \\
\hline Jobless & 0 & 0.0000 & 0.0000 & 00.00 & 0.0000 & \\
\hline \multicolumn{7}{|c|}{ Monthly income (Pakistan rupees) } \\
\hline Nil & 157 & 0.5863 & 0.2800 & 67.05 & 6.4780 & \multirow{4}{*}{0.001} \\
\hline $5000-10000$ & 2 & 0.2720 & 0.6406 & 56.00 & 8.4850 & \\
\hline $10001-15001$ & 4 & 0.7083 & 0.0669 & 65.00 & 3.8300 & \\
\hline$>15001$ & 221 & 0.6899 & 0.1736 & 66.80 & 5.2700 & \\
\hline \multicolumn{7}{|l|}{ Location } \\
\hline Urban & 220 & 0.6431 & 0.2313 & 66.33 & 5.8230 & \multirow{2}{*}{0.609} \\
\hline Rural & 164 & 0.6489 & 0.2329 & 67.49 & 5.7960 & \\
\hline \multicolumn{7}{|c|}{ Duration of disease (years) ${ }^{\star *}(3.31 \pm 2.13)$} \\
\hline$<1$ & 80 & 0.6588 & 0.2256 & 68.84 & 5.5690 & \multirow{4}{*}{0.545} \\
\hline $1-3$ & 164 & 0.6408 & 0.2361 & 67.23 & 5.6140 & \\
\hline $3-5$ & 26 & 0.6840 & 0.1912 & 66.58 & 7.6010 & \\
\hline$>5$ & $\begin{array}{l}20 \\
114\end{array}$ & 0.6343 & 0.2392 & 64.89 & 5.4050 & \\
\hline
\end{tabular}

${ }^{\star}$ Mann-Whitney test, ${ }^{\star \star}$ Kruskal-Wallis test, VAS: Visual Analogue Scale. Note: ${ }^{*}$ US $\$ 1=111.70$ Pk Rs

Table 7: Frequency of Self-reported (EQ-5D) Health States

\begin{tabular}{|c|c|c|c|c|c|c|}
\hline \multicolumn{5}{|c|}{ Health state } & \multirow[t]{2}{*}{ Frequency } & \multirow[t]{2}{*}{ (\%) } \\
\hline Mobility & Self-care & Usual activities & Pain/discomfort & Anxiety/depression & & \\
\hline 1 & 1 & 1 & 1 & 2 & 27 & 7.00 \\
\hline 1 & 1 & 1 & 1 & 3 & 6 & 1.60 \\
\hline 1 & 1 & 1 & 2 & 1 & 43 & 11.20 \\
\hline 1 & 1 & 1 & 2 & 2 & 169 & 44.00 \\
\hline 1 & 1 & 1 & 2 & 3 & 10 & 2.60 \\
\hline 2 & 1 & 1 & 1 & 2 & 1 & 0.30 \\
\hline 2 & 1 & 1 & 2 & 1 & 5 & 1.30 \\
\hline 2 & 1 & 1 & 2 & 2 & 90 & 23.40 \\
\hline 2 & 1 & 1 & 2 & 3 & 4 & 1.00 \\
\hline 2 & 1 & 1 & 3 & 2 & 1 & 0.30 \\
\hline 2 & 1 & 1 & 3 & 3 & 7 & 1.80 \\
\hline 2 & 2 & 1 & 3 & 3 & 1 & 0.30 \\
\hline 2 & 2 & 2 & 2 & 2 & 2 & 0.50 \\
\hline 2 & 2 & 2 & 2 & 3 & 4 & 1.0 \\
\hline 2 & 2 & 2 & 3 & 3 & 14 & 3.0 \\
\hline
\end{tabular}




\section{DISCUSSION}

The results from the present study showed that among the HTN patients studied, knowledge regarding hypertension had good association with medication adherence. Some studies have also reported a good relationship between hypertension-related awareness and adherence to medication $[9,10]$. It has been reported that disease-related knowledge is one of the key factors in obtaining successful adherence to therapy [11]. In contrast, a study has also reported no association between knowledge regarding hypertension and adherence to medication [12].

Reduced HRQoL was seen in the HTN patients. The poor results in HRQoL are consistent with those reported in previous studies on pulmonary and essential hypertension patients [13-15]. Nonetheless, some studies of similar nature have reported mixed results. One study reported a statistically significant relationship among education, monthly income and HRQoL [16]. In another study, it was reported that age was the only factor that showed significant relationship with HRQoL [17], while another study reported that gender and income were the only variables significantly related to HRQoL [18]. A study highlighted that age, gender, education, employment status, annual household income, obesity and hypertension were significantly associated with HRQoL [19].

In the present study, knowledge regarding hypertension had good association with HRQoL and visual analogue scale. It has been demonstrated that after counseling on lifestyle modifications, energy/fatigue scores (a quality-oflife scale) were significantly improved in hypertensive patients [20]. Moreover, it has been reported that patient education, life style modifications and motivation for health resulted in improvement in the mean score of $\mathrm{QOL}$ of hypertensive patients [21]. In another study, it was indicated that EQ-5D index and EQ-5D VAS scores of hypertensive patients were significantly improved after pharmaceutical care intervention [13]. In addition, the HRQoL of patients have been improved by promoting patient's care activities and by supporting their QoL domains [22].

Results from the present study also showed that medication adherence had good association with HRQoL and a fair correlation with visual analogue scale. In a population-based survey in Brazil, poor HRQoL was associated with lower medication adherence among hypertensive patients [23]. It was also reported that better
HRQoL was associated with higher medication adherence among patients with asthma in Japan [24]. However, another study reported a negative correlation between HRQoL and adherence in hypertensive patients [25].

A number of challenges are faced by developing countries in attempts to provide optimal healthcare to their citizens. Within this context, Pakistan is the $6^{\text {th }}$ most populous country in the world, with about 40 million people living below national poverty line, and half of the adult population is illiterate. More important is the fact that across regions, disparities in income per capita have persisted or even widened [22].

It is possible to attain optimized pharmaceutical care through provision of education to patients regarding self-management. This will result in improvement of their knowledge and understanding about hypertension, adherence to therapy, and coping strategies. The present research revealed that a large portion of patients had poor knowledge of hypertension, and had little information regarding management and control of the disease.

\section{Limitations of the study}

In current study, majority of hypertensive patients were in the age range of 41-50 years, and above that age majority of them had comorbidities like diabetes which limited the number of patients that met the inclusion criteria for this study. Therefore, the results of the current study cannot be generalized. .

\section{CONCLUSION}

The results obtained in this study show that knowledge about hypertension has a good association with medication adherence and HRQoL. A good association also exists between medication adherence and HRQoL.

\section{DECLARATIONS}

\section{Acknowledgement}

The authors acknowledge all the hypertensive patients enrolled in current study and the staff of the hospital for their support in successfully accomplishing the study.

\section{Conflict of interest}

No conflict of interest is associated with this work. 


\section{Contribution of authors}

We declare that this work was done by the authors named in this article and all liabilities pertaining to claims relating to the content of this article will be borne by the authors.

\section{Open Access}

This is an Open Access article that uses a funding model which does not charge readers or their institutions for access and distributed under the terms of the Creative Commons Attribution License (http://creativecommons.org/licenses/by/ 4.0) and the Budapest Open Access Initiative (http://www.budapestopenaccessinitiative.org/rea d), which permit unrestricted use, distribution, and reproduction in any medium, provided the original work is properly credited.

\section{REFERENCES}

1. Saleem F, Hassali M, Shafie AA, Awad A, Bashir S. Association between knowledge and drug adherence in patients with hypertension in Quetta, Pakistan. Trop $J$ Pharm Res 2011; 10(2).

2. Van Lerberghe W, Manuel A, Matthews Z, Cathy W. The World Health Report 2005-make every mother and child count: World Health Organization, 2005.

3. Akoko BM, Fon PN, Ngu RC, Ngu KB. Knowledge of hypertension and compliance with therapy among hypertensive patients in the Bamenda Health District of Cameroon: a cross-sectional study. Cardiol Therap 2017; 6(1): 53-67.

4. Roca B, Nadal E, Rovira RE, Valls S, Lapuebla C, Lloria $N$. Usefulness of a hypertension education program. South Med J 2003; 96(11): 1133-1138.

5. Nazir SUR, Hassali MA, Saleem F, Bashir S, Aljadhey H. Association Between Diabetes-related Knowledge and Medication Adherence: Results From Cross-sectional Analysis. Altern Ther Health Med 2016; 22(6): 8-13.

6. EuroQol G. EuroQol--a new facility for the measurement of health-related quality of life. Health policy (Amsterdam, Netherlands) 1990; 16(3): 199.

7. Shaw JW, Johnson JA, Coons SJ. US valuation of the $E Q-5 D$ health states: development and testing of the D1 valuation model. Med Care 2005; 43(3): 203-220.

8. Dolan $P$, Gudex $C$, Kind $P$, Williams $A$. A social tariff for EuroQol: results from a UK general population survey 1995.

9. Kjellgren Kl, AhIner J, Säljö R. Taking antihypertensive medication-controlling or co-operating with patients? Int J Cardiol 1995; 47(3): 257-268.

10. Orth JE, Stiles WB, Scherwitz L, Hennrikus D, Vallbona C. Patient exposition and provider explanation in routine interviews and hypertensive patients' blood pressure control. Health Psychol 1987; 6(1): 29.
11. Heurtin-Roberts S, Reisin E. The relation of culturally influenced lay models of hypertension to compliance with treatment. Am J Hypertens 1992; 5(11): 787-792.

12. Haynes RB, Gibson E, Hackett B, Sackett D, Taylor DW, Roberts $R$, Johnson $A$. Improvement of medication compliance in uncontrolled hypertension. Lancet 1976; 307 (7972): 1265-1268.

13. Akhtar MM, Waheed A, Sheikh D, Hussain A. Role of pharmacist in improving health related quality of life (HRQoL) in hypertensive patients in Pakistan. Am J Pharmacol Sci 2014; 2 (5B): 17-22.

14. Taichman DB, Shin J, Hud L, Archer-Chicko C, Kaplan S, Sager JS, Gallop R, Christie J, Hansen-Flaschen J, Palevsky $\mathrm{H}$. Health-related quality of life in patients with pulmonary arterial hypertension. Respir Res 2005; 6(1): 92.

15. Mena-Martin FJ, Martin-Escudero JC, Simal-Blanco F, Carretero-Ares JL, Arzua-Mouronte D, HerrerosFernandez V. Health-related quality of life of subjects with known and unknown hypertension: results from the population-based Hortega study. J Hypertens 2003; 21(7): 1283-1289.

16. Khosravi A, Arash Ramezani M, Toghianifar N, Rabiei K, Jahandideh $M$, Yousofi A. Association between hypertension and quality of life in a sample of Iranian adults. Acta Cardiol 2010; 65(4): 425-430.

17. Pappa E, Kontodimopoulos N, Papadopoulos AA, Niakas $D$. Assessing the socio-economic and demographic impact on health-related quality of life: evidence from Greece. Int J Public Health 2009; 54(4): 241-249.

18. Baune B, Aljeesh Y. The association of psychological stress and health related quality of life among patients with stroke and hypertension in Gaza Strip. Ann Gen Psychiatry 2006; 5(1): 6.

19. Goins RT, John R, Hennessy CH, Denny CH, Buchwald $D$. Determinants of health-related quality of life among older American Indians and Alaska Natives. J Appl Gerontol 2006; 25 (1 Suppl): 73S-88S.

20. Park JJ, Kelly P, Carter BL, Burgess PP. Comprehensive Pharmaceutical Care in the Chain Setting: Drug therapy monitoring and counseling by pharmacists contributed to improved blood pressure control in study patients. J Am Pharm Assoc 1996; 36(7): 443-451.

21. Wal $P$, Wal A, Bhandari A, Pandey $U$, Rai $A K$. Pharmacist involvement in the patient care improves outcome in hypertension patients. J Res Pharm Pract 2013; 2(3): 123.

22. Saleem F, Hassali MA, Shafie AA. A cross-sectional assessment of health-related quality of life (HRQoL) among hypertensive patients in Pakistan. Health Expect 2014; 17(3): 388-395.

23. Trevisol D, Moreira L, Fuchs F, Fuchs S. Health-related quality of life is worse in individuals with hypertension under drug treatment: results of population-based study. $J$ Hum Hypertens 2012; 26(6): 374.

24. Takemura M, Kobayashi M, Kimura K, Mitsui K, Masui $H$, Koyama M, Itotani R, Ishitoko M, Suzuki S, Aihara K. Repeated instruction on inhalation technique improves 
adherence to the therapeutic regimen in asthma. $J$ Asthma 2010; 47(2): 202-208.

25. Saleem F, Hassali MA, Shafie AA, Awad GA, Atif M, ul Haq $N$, Aljadhey $H$, Farooqui $M$. Does treatment adherence correlates with health related quality of life? Findings from a cross sectional study. BMC Public Health 2012; 12(1): 318. 